\title{
ARTICLE
}

Clinical Study

\section{Plasma total cell-free DNA is a prognostic biomarker of overall survival in metastatic solid tumour patients}

\author{
Ida Viller Tuxen ${ }^{1}$, Lise Barlebo Ahlborn ${ }^{1,2}$, Morten Mau-Soerensen ${ }^{1}$, Kristoffer Staal Rohrberg ${ }^{1}$, Finn Cilius Nielsen ${ }^{2}$, Olga Oestrup ${ }^{2}$, \\ Christina Westmose Yde $^{2}$, Ivan Richter Vogelius ${ }^{3}$ and Ulrik Lassen ${ }^{1}$
}

BACKGROUND: Selecting patients for early clinical trials is a challenging process and clinicians lack sufficient tools to predict overall survival (OS). Circulating cell-free DNA (cfDNA) has recently been shown to be a promising prognostic biomarker. The aim of this study was to investigate whether baseline cfDNA measurement could improve the prognostic information of the Royal Marsden Hospital (RMH) score.

METHODS: Solid tumour patients referred for phase I trials were included in the Copenhagen Personalized Oncology (CoPPO) programme. Baseline characteristics were collected prospectively, including the RMH prognostic score, Eastern Cooperative Oncology Group (ECOG) performance status and concentration of cfDNA per millilitre plasma. Cox proportional hazards model was used to assess the prognostic value of baseline variables.

RESULTS: Plasma cfDNA concentration was quantifiable in 302 patients out of a total of 419 included in the study period of 2 years and 5 months. The RMH score was confirmed to be associated with OS. Cell-free DNA was shown to be an independent prognostic marker of OS and improved the risk model, including $\mathrm{RMH}$, performance status and age. Furthermore, both plasma cfDNA concentration and RMH score were associated with treatment allocation $(p<0.00001)$.

CONCLUSION: Our model based on RMH score, age, ECOG performance status and cfDNA improved prediction of OS and constitutes a clinically valuable tool when selecting patients for early clinical trials. An interactive version of the prognostic model is published on http://bit.ly/phase1survival.

British Journal of Cancer (2019) 121:125-130; https://doi.org/10.1038/s41416-019-0491-9

\section{BACKGROUND}

Cancer patients with exhausted treatment options can be referred to phase 1 clinical trials where the primary objectives are dose finding and toxicity assessment. However, only a minority of patients will benefit from treatment ${ }^{1,2}$ and selection of the most appropriate patient for therapy is challenging. Generally used eligibility criteria include adequate organ function, good performance status and life expectancy more than 3 months. Clinicians often misjudge the survival of the patients leading to screen failures and waste of important time for the individual patient. ${ }^{3-5}$

Different prognostic scores have been introduced in order to select patients for phase 1 trials. ${ }^{6,7}$ The Royal Marsden Hospital $(\mathrm{RMH})$ score has been validated in various phase 1 cohorts $^{8-11}$ and consist of three variables: Elevated lactate dehydrogenase (LDH) (> upper limit), low serum-albumin $(<35 \mathrm{~g} / \mathrm{L})$ and more than two metastatic sites. Although, these prognostic scores have been validated, the clinical use is limited.

In recent years, much attention in oncological studies has been given to circulating cell-free DNA (cfDNA) as a non-invasive tumour marker used in diagnostics and treatment monitoring. Cell-free DNA is short fragments of DNA present in plasma and other body fluids and originates from apoptotic and necrotic cells representing normal tissue and potentially multiple tumour lesions. ${ }^{12}$ cfDNA has been highlighted as a new potential biomarker for overall survival (OS) in various cancer subtypes. ${ }^{13-15}$ To investigate the clinical utility of cfDNA in patients referred to phase 1 trials, we conducted a pre-planned examination of baseline plasma cfDNA levels in patients participating in the Copenhagen Prospective Personalized Oncology (CoPPO) trial. ${ }^{16}$ The aim of the study was to investigate whether additional measurements of plasma cfDNA concentration improved the prognostic value of the $\mathrm{RMH}$ score and thus selection of patients for phase I trials.

\section{METHODS}

Patients and study design

This study included patients enrolled in the CoPPO study (NCT02290522) from October 2014-February 2017. The CoPPO study aims to investigate the clinical utility of molecular profiling to select patients for early clinical trials. All patients fulfilled the inclusion criteria including: exhausted treatment options, life expectancy $\geq 3$ months, normal organ function, age $\geq 18$ years, Eastern Cooperative Oncology Group (ECOG) performance status 0 or 1 and lesions assessable for biopsy. Basic characteristics

\footnotetext{
${ }^{1}$ The Phase I Unit, Department of Oncology, Copenhagen University, Rigshospitalet, Copenhagen, Denmark; ${ }^{2}$ Center for Genomic Medicine, Copenhagen University, Rigshospitalet, Copenhagen, Denmark and ${ }^{3}$ Section of Radiotherapy, Department of Oncology, Copenhagen University, Rigshospitalet, Copenhagen, Denmark Correspondence: Ulrik Lassen (Ulrik.lassen@regionh.dk)

These authors contributed equally: Ida Viller Tuxen, Lise Barlebo Ahlborn, Ivan Richter Vogelius, Ulrik Lassen
}

Received: 26 November 2018 Revised: 9 May 2019 Accepted: 16 May 2019

Published online: 12 June 2019 
including serum albumin and lactate dehydrogenase (LDH) were registered at inclusion. Values obtained within 2 months of inclusion were accepted.

The study was conducted in accordance with the Declaration of Helsinki and approved by the Danish Data Protection Agency and the Regional Ethics Committee (Danish Ethical Committee, file number: 1300530). All patients provided signed informed consent. Cut-off date was 22 February 2018. Examination of plasma cfDNA concentration was planned but the statistical analysis was not pre-specified and thereby considered as exploratory.

Extraction and quantification of cfDNA

Peripheral blood was collected in cell-stabilising Blood Collection Tubes (BCT; Streck Laboratories, Omaha, NE, USA) and cfDNA was extracted from 2-4 millilitre $(\mathrm{ml})$ plasma using the QIAsymphony Circulating DNA Kit (Qiagen, Hilden, Germany) according to the manufacturer's instructions using an elution volume of $60 \mu \mathrm{l}$ as previously described. ${ }^{17}$ Quantification of cfDNA was performed using the dsDNA HS Assay Kit ( $>10 \mathrm{pg} / \mu \mathrm{L}$ ) on a Qubit Fluorometer (Thermo Fisher Scientific, Waltham, MA) and the concentration of cfDNA per $\mathrm{ml}$ of plasma was calculated for each sample. ${ }^{18}$

For validation of the cfDNA quantification method, we measured total cfDNA in triplicates using the Agilent 4200 TapeStation system (D5000) for 49 samples with low cfDNA concentration $(0-15 \mathrm{ng} / \mathrm{ml}$ plasma (A)) and 47 samples with high concentration $(50-1000 \mathrm{ng} / \mathrm{ml}$ plasma (B)) (Fig. S2). For the high-concentration group we diluted samples $(1: 10)$ if the concentration was $>100 \mathrm{ng} / \mathrm{ml}$ plasma. The Agilent 4200 TapeStation system (D5000) uses electrophoresis to separate DNA fragments from $100-5000 \mathrm{bp}$ and can quantify DNA concentrations down to $0.1 \mathrm{ng} / \mu \mathrm{L}$. In contrast, the Qubit system uses intercalating fluorescent dyes binding only double stranded DNA, leading to concentration measures around half the value of the ones from the TapeStation. This was supported by the Bland-Altman plot shown in Fig. S3 illustrating the concordance between Qubit and TapeStation cfDNA quantification (mean percentage difference between the methods of $21.8 \%$ and limits of agreement around $\pm 40 \%$ ).

\section{Statistical analysis}

OS (Kaplan-Meier) was calculated from the date of inclusion to time of death or censure. The prognostic value of the RMH score was tested using a univariate Cox model with $\mathrm{RMH}$ score as scale input. Subsequently, multivariable analysis was performed with performance status and RMH score as predefined categorical covariables. Age and the log-transformed value of cfDNA (logcfDNA) from the Qubit system was included as continuous variables. Coding (e.g. logcfDNA) was confirmed by comparison with restricted cubic spines.

Correlations between the covariables $\mathrm{RMH}$ score, age and logcfDNA were assessed visually and the validity of the proportional hazards assumption on performance status and RMH score was investigated by visual assessment of log minus-log survival curves. Statistical analysis was performed in $\mathrm{R}$ using the cphfunction of the RMS package. ${ }^{19}$ In all cases a $p$-value of 0.05 or less was considered significant. The final model is published as an interactive version using the Shiny package in $\mathrm{R}^{20}$ We tested a possible association between cfDNA (quartiles)/RMH score and the risk of not being offered treatment using logistic regression by $\mathrm{glm}$ package in $\mathrm{R}$ treating both cfDNA quartiles (25th percentile: $7.4 \mathrm{ng} / \mathrm{ml}$; 50 th percentile: $17.1 \mathrm{ng} / \mathrm{ml}$; 75 th percentile: $39.55 \mathrm{ng} /$ $\mathrm{ml}$ ) and $\mathrm{RMH}$ score as a numerical covariate.

\section{RESULTS}

Patient characteristics

A total of 419 patients were included in the CoPPO study within the study period. Measurable plasma cfDNA concentration at inclusion was obtained from 302 patients and 169 patients were allocated to treatment and 133 received no further treatment (Fig. 1).

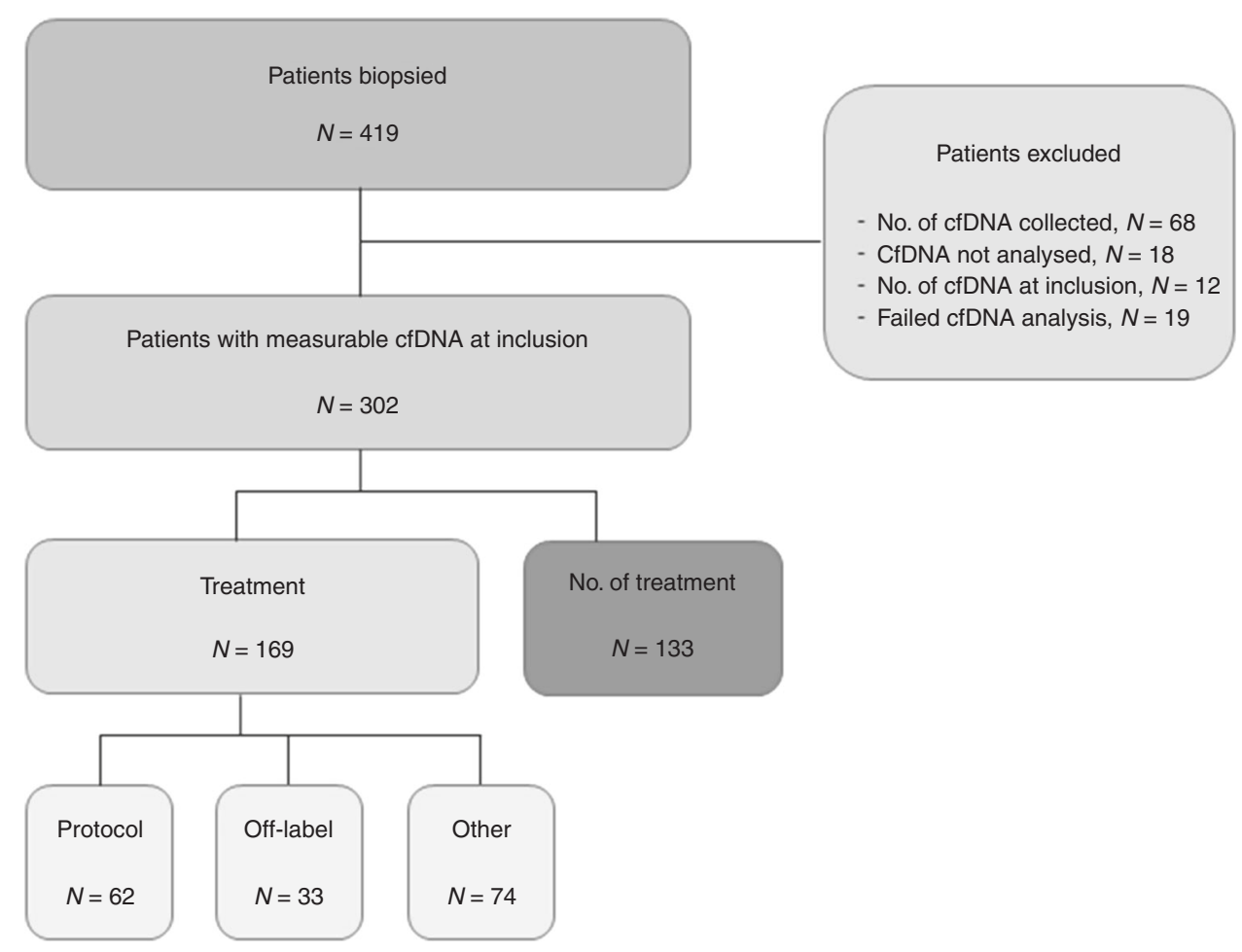

Fig. 1 Patient flowchart. A total of 419 patients were included within the study time (3 October 2014 to 22 February 2017 ) including 302 patients with available plasma cfDNA samples collected at the time of tissue biopsy (baseline). A total of 169 patients were allocated to treatment whereas 133 patients received no further treatment 
Baseline patient characteristics are summarised in Table 1. There was an equal distribution of gender and age. Patients had received a median of three prior treatment regimens and the majority of patients $(63 \%)$ presented with more than two metastatic sites. Baseline LDH was elevated in 196 patients (65\%) and 168 patients $(56 \%)$ presented with serum-albumin below normal level. In total, one third of the patients (32\%) were classified as having a good prognostic score (RMH score $0-1)$ with most patients having a performance score of $1(66 \%)$ according to ECOG guidelines. Median OS was 24 weeks (95\% Cl 21.0-27.0) and median follow-up time of censored cases $(n=35)$ was 84 weeks (Cl 73.6-94.4). The most common tumour types were colorectal cancer $(25 \%)$, followed by breast $(12 \%)$, pancreatic $(9 \%)$ and bile duct cancers (8\%) (Table S1). Plasma concentration of cfDNA according to cancer subtype is reported in Fig. 2 with an overall median of $17.1 \mathrm{ng} / \mathrm{ml}(1.1-795.0)$ (Table S1). Both RMH score and cfDNA quartile was highly associated with the probability of not being offered treatment (Fig. 3, $P<0.00001$ for both RMH score and (fDNA).

Prognostic value of cfDNA on survival

Univariate survival analysis. We confirmed a clear association between the RMH score and OS (log-rank $P<0.0001$ ) (Fig. 4a). Univariate survival for the four quartiles of cfDNA showed a significant association between cfDNA levels and OS (log-rank $P<0.0001$ ) (Fig. 4b). No correlations were observed between $\mathrm{RMH}$ score, performance status and the age of the patients. Furthermore, there was no correlation between RMH score and cfDNA (Fig. S1).

Multivariate survival analysis. In multivariate analysis including age, RMH, performance status and logcfDNA, low plasma concentration of cfDNA was associated with longer OS with a
Table 1. Patient characteristics

\begin{tabular}{|c|c|}
\hline & Total \\
\hline & $(n=302)$ \\
\hline \multicolumn{2}{|l|}{ Gender } \\
\hline Female & $145(48 \%)$ \\
\hline Male & $157(52 \%)$ \\
\hline \multicolumn{2}{|l|}{ Age } \\
\hline Median, range & $60(26-86)$ \\
\hline \multicolumn{2}{|l|}{ Metastatic sites } \\
\hline$\leq 2$ & $111(37 \%)$ \\
\hline$>2$ & $190(63 \%)$ \\
\hline Missing & $1(0 \%)$ \\
\hline \multicolumn{2}{|l|}{ Albumin } \\
\hline$\geq 35 \mathrm{~g} / \mathrm{L}$ & $129(43 \%)$ \\
\hline$<35 \mathrm{~g} / \mathrm{L}$ & $168(56 \%)$ \\
\hline Missing & $5(2 \%)$ \\
\hline \multicolumn{2}{|l|}{$L D H$} \\
\hline$>$ ULN & $196(65 \%)$ \\
\hline$\leq \mathrm{ULN}$ & $104(34 \%)$ \\
\hline Missing & $2(1 \%)$ \\
\hline \multicolumn{2}{|l|}{ RMH score } \\
\hline $0-1$ & $97(32 \%)$ \\
\hline $2-3$ & $200(66 \%)$ \\
\hline Missing & $5(2 \%)$ \\
\hline \multicolumn{2}{|c|}{ Number of prior treatment regimens } \\
\hline Median, range & $3(1-11)$ \\
\hline \multicolumn{2}{|c|}{ Performance status (ECOG) } \\
\hline 0 & $99(33 \%)$ \\
\hline 1 & $200(66 \%)$ \\
\hline 2 & $3^{*}(1 \%)$ \\
\hline
\end{tabular}

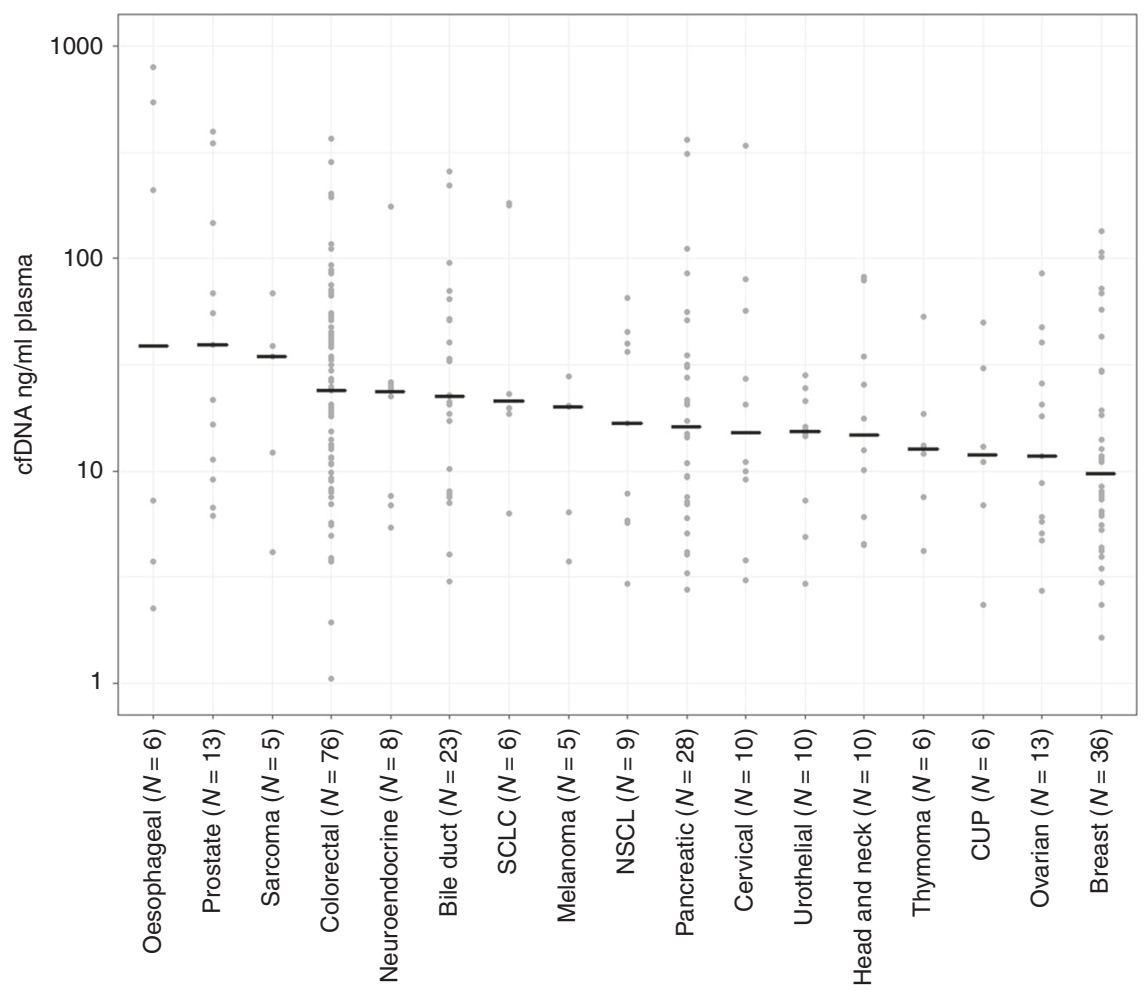

Fig. 2 Distribution of cfDNA concentrations ( $\mathrm{ng} / \mathrm{ml}$ plasma) in diverse cancer subtypes. Cancer types including five or more patients are indicated with cfDNA concentrations and the median value for each subtype is indicated by a horizontal line. Each dot represents a patient included in the study 

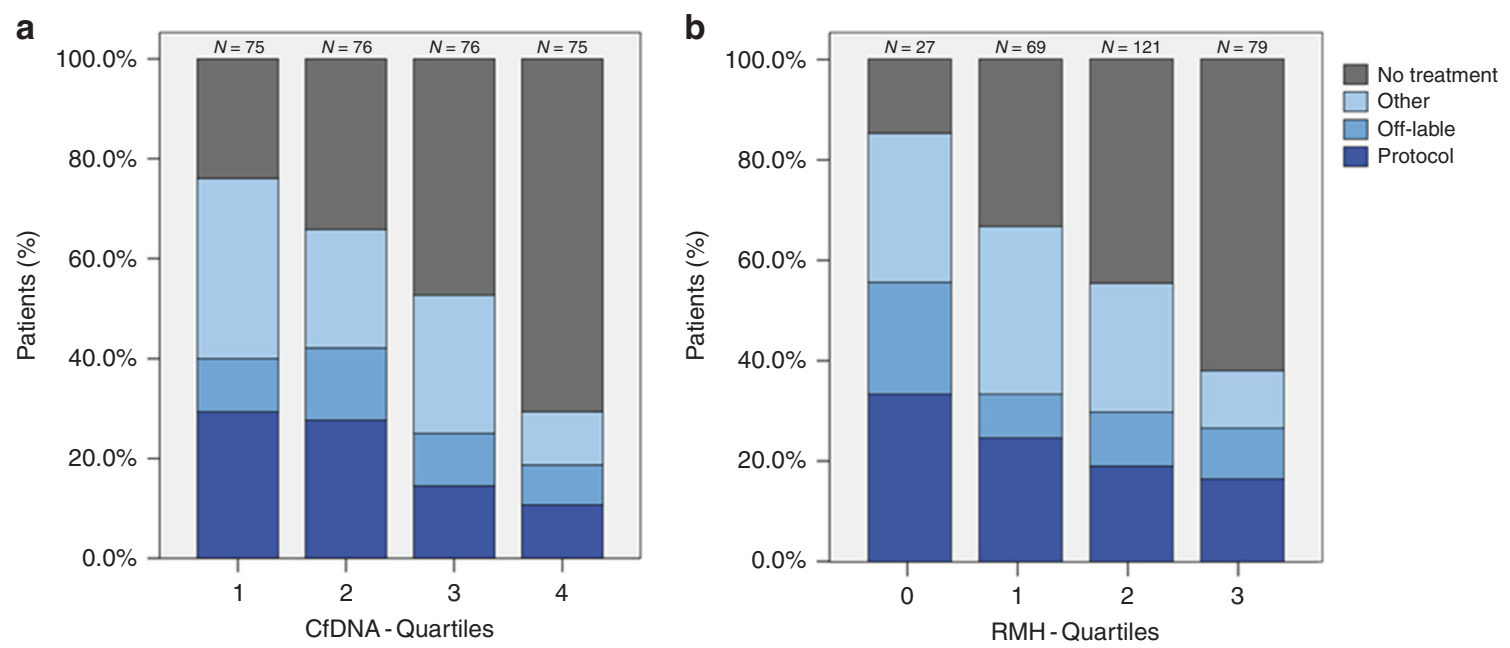

Fig. 3 Treatment allocation based on cfDNA concentration (a) and RMH score (b). The percentage of patients allocated to further treatment or not are indicated based on either cfDNA quantiles ( 25 percentile: $7.4 \mathrm{ng} / \mathrm{ml} ; 50$ percentiles: $17.1 \mathrm{ng} / \mathrm{ml} ; 75$ percentiles: $39.55 \mathrm{ng} / \mathrm{ml}$ ) or RMH score (0-3). Treatment allocation included treatments in a non-phase 1 setting ("Other"), off-label treatment with approved therapy in other diagnosis ("Off-label") and patients in a phase 1 protocol treatment ("Protocol"). $N$ indicates the number of patients in each group. Note that patients are equally distributed in cfDNA quantiles (a) in contrast to RMH score (b)

hazard ratio (HR) of 1.67 (95\% Cl: $1.39-2.01 ; P<0.0001)$. No association between age and survival was observed (Table 2).

The OS curves variate according to altering concentrations of cfDNA although RMH score remains the same. An interactive version of the prognostic model is published on http://bit.ly/ phase1survival and presented in Fig. S4 by a typical patient from the cohort aged 60 years, $\mathrm{RMH}$ score $=2$, performance status $=1$ and cfDNA levels of 20 and $100 \mathrm{ng} / \mathrm{ml}$ plasma.

\section{DISCUSSION}

Clinicians treating patients with exhausted treatment options are facing the dilemma of choosing when to refer the patient to early clinical trials. The RMH score can be used as a prognostic score to guide this decision. In this study, we have shown that by adding the baseline measurement of plasma cfDNA concentration, performance status and age to the RMH score, we were able to build a stronger prediction model assessing OS. This model showed superiority compared to RMH score alone. An improved risk assessment could support the clinical decision when enrolling patients in phase I studies as early discontinuation of patients in phase 1 trials most frequently are due to progression and clinical deteoriation. ${ }^{21}$ The clinical implementation of the proposed model therefore has the potential to improve selection of patients to phase I trials. Furthermore, low plasma concentration of cfDNA at baseline is correlated with a higher chance of receiving further treatment. Similar results were observed with $\mathrm{RMH}$ score, suggesting that both markers could be valuable tools in allocating patients to treatment in a phase 1 setting.

Our patient cohort represented a consecutive group of patients included in a molecular profiling project with most patients not receiving treatment within a clinical trial (Fig. 3). It seems likely, that this cohort is representative for a typical cohort of patients referred to phase 1 trials.

However, there are limitations to this study; this is a single centre study and the risk model requires validation in other phase 1 cohorts. Furthermore, we could not exclude whether treatment affected the survival analysis. Low concentrations of cfDNA $(<5 \mathrm{ng} / \mathrm{ml})$ can be measured in healthy individuals mostly emerging from haematopoietic cells $s^{22}$ and the concentration of cfDNA can be influenced by other factors including inflammation, infectious disease, exercise etc. These are all confounders of our model and must be considered. For clinical use we would recommend the blood samples to be collected without prior physical activity and notion of an infection. In addition, we only used one method to quantify the cfDNA concentrations. The fluorescence-based Qubit HS assay was selected as this is a simple, fast, cheap and standard method used in most clinical laboratories. Similar assays using intercalating fluorophores have been widely used to detect low levels of $\mathrm{cfDNA}^{18,23}$ but other methods are being validated to optimise the accuracy of cfDNA quantification including digital and real-time PCR. ${ }^{24,25}$ Larger studies comparing the different methods are needed to standardise the quantification of cfDNA.

Total cfDNA have previously been shown as a prognostic factor in selected cancer types ${ }^{13-15}$ but, to our knowledge, never used to predict survival in the phase 1 setting across a wide range of cancer types. Additionally, cfDNA has never been added to the $\mathrm{RMH}$ score and our data support the addition of cfDNA to improve prediction of survival compared to $\mathrm{RMH}$ alone. Olmos et al. have shown that adding the number of circulating tumour cells (CTCS) to the RMH score, improved estimation of OS. ${ }^{26}$ However, this has not been implemented in clinical practice, probably due to the laborious process of isolating CTCs.

In conclusion, we present a model based on a singlecentre study, that offers a valuable supplement to the $\mathrm{RMH}$ score providing a further improved estimation of the OS for the individual patient. This study supports the continuing efforts exploring the clinical role of cfDNA in cancer care.

\section{ACKNOWLEDGEMENTS}

We sincerely thank patients and staff at the Phase I unit and Centre for Genomic Medicine, Copenhagen University, Rigshospitalet.

\section{AUTHOR CONTRIBUTIONS}

The study was designed by I.V.T. and L.B.A. who also collected the clinical and experimental data and wrote the paper. I.R.V. performed the statistical analyses. K.S.R. and M.M-S. assisted in collecting the patient information and enrolled patients in the CoPPO study headed by F.C.N. and U.L. O.Ø. and C.W.Y. supported the experimental work of cfDNA quantification. 
a

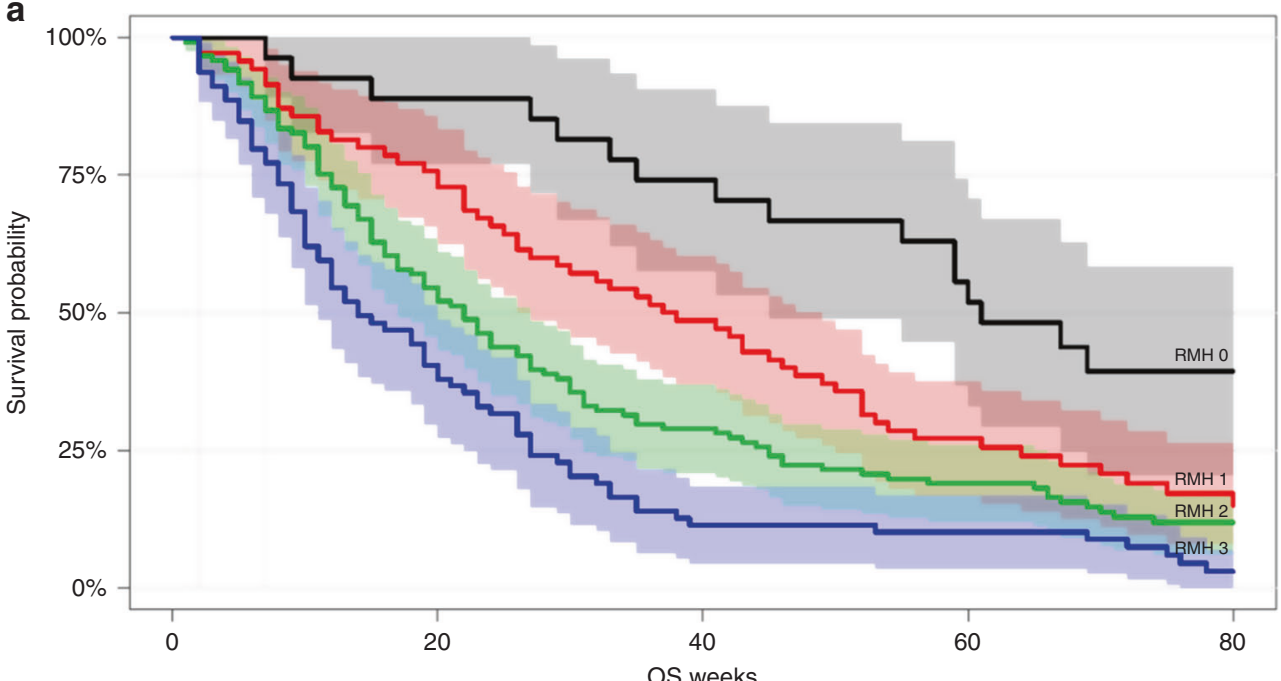

$\begin{array}{rccccccccccc}\text { RMH } & & & & & & & & & & & \\ 0: & 27 & 26 & 24 & 24 & 22 & 20 & 18 & 17 & 12 & 8 & 6 \\ 1: & 70 & 64 & 56 & 47 & 40 & 34 & 27 & 20 & 16 & 12 & 8 \\ 2: & 121 & 105 & 76 & 56 & 40 & 35 & 27 & 24 & 22 & 14 & 11 \\ 3: & 79 & 61 & 38 & 26 & 16 & 9 & 9 & 8 & 8 & 6 & 2\end{array}$

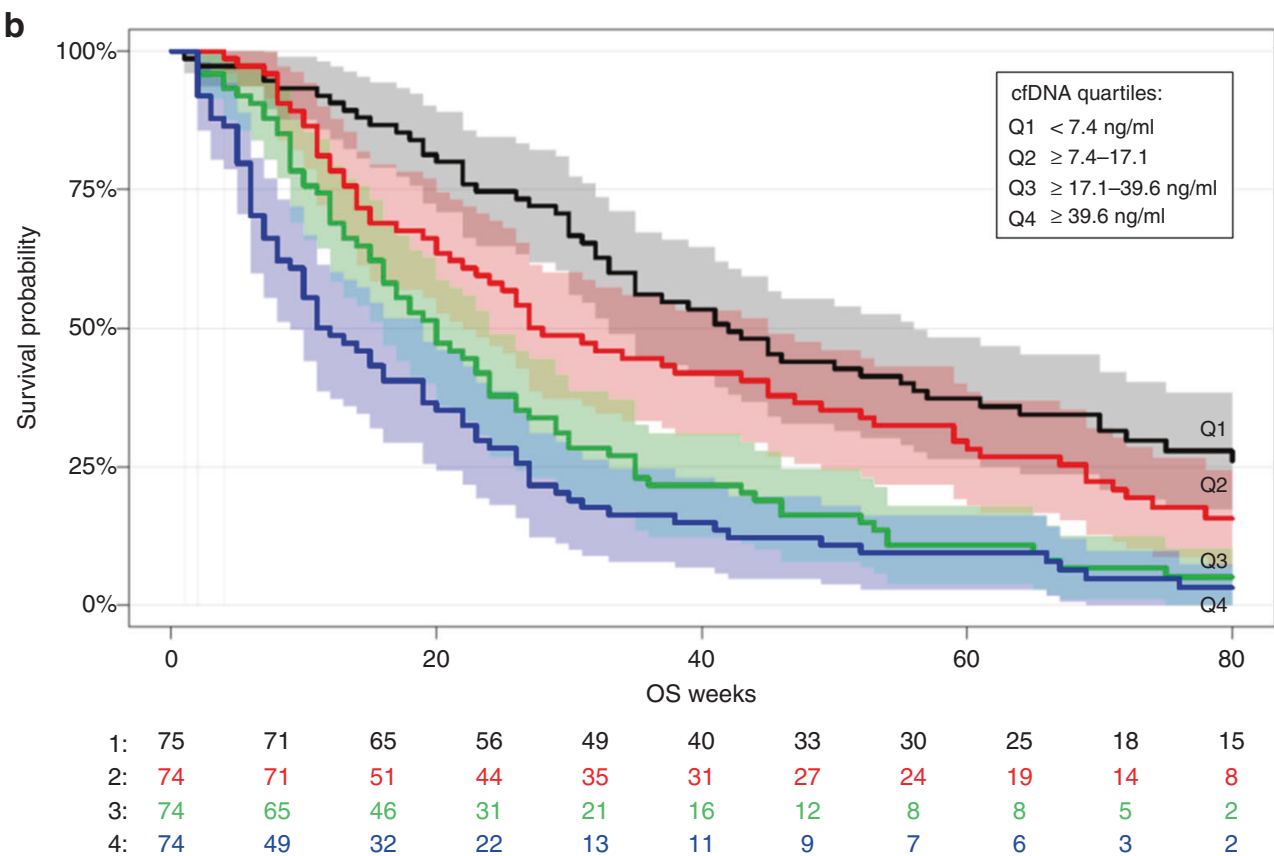

Fig. 4 Survival analysis according to RMH score (a) and cfDNA level quantiles (b). A total of 297 patients were included in the survival analysis. The five patients excluded from the analysis where patients without RMH score (Table 1, patients marked with *). Cell-free DNA levels were split in quartiles

\begin{tabular}{|llc|}
\hline Table 2. Cox proportional hazards model & \\
\hline & Hazard ratio $(95 \% \mathrm{Cl})$ & $P$-value \\
\hline Age & $0.97(0.82-1.16)$ & 0.77 \\
cfDNA [log] & $1.67(1.39-2.01)$ & $<0.0001$ \\
RMHI 1 vs. 0 & $2.08(1.22-3.57)$ & 0.008 \\
RMHI 2 vs. 0 & $2.29(1.38-3.80)$ & 0.001 \\
RMHI 3 vs. 0 & $3.57(2.12-6.03)$ & $<0.0001$ \\
PS 1 vs. 0 & $2.35(1.77-3.13)$ & $<0.0001$ \\
\hline
\end{tabular}

\section{ADDITIONAL INFORMATION}

Supplementary information is available for this paper at https://doi.org/10.1038/ s41416-019-0491-9.

Competing interests: M.M-S. has received lecture honoraria and support to participate in the scientific conference from Roche. K.S.R. received support to participate in the scientific conference from Roche and Sanofi. The remaining authors declare no competing interests.

Ethics approval and consent to participate: Regulatory approvals from the Regional Ethics Committee and the Danish Data Protection Agency were obtained (Danish Ethical Committee, file number: 1300530). All patients provided signed 
informed consent and the study was performed in accordance with the Declaration of Helsinki.

Funding: We thank the Capital Region of Copenhagen and the Oncological Research Fund, Department of Oncology, Copenhagen University Hospital, Denmark for financial support.

Data availability: Data is available as Supplementary material. An interactive version of the prognostic model is published on http://bit.ly/phase1survival

Note: This work is published under the standard license to publish agreement. After 12 months the work will become freely available and the license terms will switch to a Creative Commons Attribution 4.0 International (CC BY 4.0).

Publisher's note: Springer Nature remains neutral with regard to jurisdictional claims in published maps and institutional affiliations.

\section{REFERENCES}

1. Horstmann, E., McCabe, M. S., Grochow, L., Yamamoto, S., Rubinstein, L., Budd, T. et al. Risks and benefits of phase 1 oncology trials, 1991 through 2002. N. Engl. J. Med. 352, 895-904 (2005)

2. Schwaederle, M., Zhao, M., Jack Lee, J., Lazar, V., Leyland-Jones, B., Schilsky, R. L. et al. Association of biomarker-based treatment strategies with response rates and progression-free survival in refractory malignant neoplasms a meta-analysis. JAMA Oncol. 2, 1452-1459 (2016).

3. Bachelot T. D., Catimel G., Ardiet C., Guastalla J. P., Dumortier A., Chauvin F. et al. Multivariable analysis of prognostic factors for toxicity and survival for patients enrolled in phase I clinical trials. Ann Oncol. 11, 151-156 (2000)

4. Ploquin, A., Olmos, D., Ferté, C., Cassier, P. A., Kramar, A., Duhamel, A. et al. Lifeexpectancy of patients enrolled in phase 1 clinical trials: a systematic review of published prognostic models. Crit. Rev. Oncol. Hematol. 83, 242-248 (2012).

5. Ploquin, A., Olmos, D., Lacombe, D., A'Hern, R., Duhamel, A., Twelves, C. et al. Prediction of early death among patients enrolled in phase I trials: development and validation of a new model based on platelet count and albumin. Br. J. Cancer 107, 1025-1030 (2012).

6. Arkenau, H.-T., Barriuso, J., Olmos, D., Ang, J. E., de Bono, J., Judson, I. et al. Prospective validation of a prognostic score to improve patient selection for oncology phase I trials. J. Clin. Oncol. 27, 2692-2696 (2009).

7. Wheler, J., Tsimberidou, A. M., Hong, D., Naing, A., Falchook, G., Piha-Paul, S. et al. Survival of 1,181 patients in a phase I clinic: the MD Anderson Clinical Center for targeted therapy experience. Clin. Cancer Res. 18, 2922-2929 (2012).

8. Arkenau, H. T., Olmos, D., Ang, J. E., De Bono, J., Judson, I. \& Kaye, S. Clinical outcome and prognostic factors for patients treated within the context of a phase I study: the Royal Marsden Hospital experience. Br. J. Cancer 98, 1029-1033 (2008).

9. Chau, I., Ashley, S. \& Cunningham, D. Validation of the Royal Marsden hospital prognostic index in advanced esophagogastric cancer using individual patient data from the REAL 2 study. J. Clin. Oncol. 27, e3-e4 (2009).

10. Garrido-Laguna, I., Janku, F., Vaklavas, C., Falchook, G. S., Fu, S., Hong, D. S. et al. Validation of the Royal Marsden Hospital prognostic score in patients treated in the phase I clinical trials program at the MD Anderson Cancer Center. Cancer 118, 1422-1428 (2012).
11. Livingston, J. A., Hess, K. R., Naing, A., Hong, D. S., Patel, S., Benjamin, R. S. et al. Validation of prognostic scoring and assessment of clinical benefit for patients with bone sarcomas enrolled in phase I clinical trials. Oncotarget 7, 64421-64430 (2016).

12. Jahr, S., Hentze, H., Englisch, S., Hardt, D., Fackelmayer, F. O., Hesch, R. D. et al. DNA fragments in the blood plasma of cancer patients: quantitations and evidence for their origin from apoptotic and necrotic cells. Cancer Res. 61, 1659-1665 (2001).

13. Cheng, J., Holland-Letz, T., Wallwiener, M., Surowy, H., Cuk, K., Schott, S. et al. Circulating free DNA integrity and concentration as independent prognostic markers in metastatic breast cancer. Breast Cancer Res. Treat. 169, 69-82 (2018).

14. Valpione, S., Gremel, G., Mundra, P., Middlehurst, P., Galvani, E., Girotti, M. R. et al. Plasma total cell-free DNA (cfDNA) is a surrogate biomarker for tumour burden and a prognostic biomarker for survival in metastatic melanoma patients. Eur. J. Cancer 88, 1-9 (2018).

15. Mehra N., Dolling D., Sumanasuriya S., Christova R., Pope L., Carreira S., et al. Plasma cell-free DNA concentration and outcomes from Taxane therapy in metastatic castration-resistant prostate cancer from two phase III trials (FIRSTANA and PROSELICA). Eur. Urol. 74, 283-291 (2018)

16. Tuxen I. V., Santoni-Rugiu E., Joenson Lars, Vikesaa J., Mau-Sørensen M., Nielsen F. $C$., et al. Copenhagen prospective personalized oncology (CoPPO): sequencing and array-based pipeline for selection of patients to phase 1 studies. J Clin. Oncol. 32, 11097 (2014).

17. Ahlborn, L. B., Madsen, M., Joenson, L., Nielsen, F. C., Lassen, U., Yde, C. W. et al. Concordance of mutation detection in circulating tumor DNA in early clinical trials using different blood collection protocols. Clin. Lab. 63, 1755-1759 (2017).

18. Kloten, V., Rüchel, N., Brüchle, N. O., Gasthaus, J., Freudenmacher, N., Steib, F. et al. Liquid biopsy in colon cancer: comparison of different circulating DNA extraction systems following absolute quantification of KRAS mutations using Intplex allele-specific PCR. Oncotarget 8, 86253-86263 (2017). 21.

19. Harrel F. E. rms: Regression Modeling Strategies. https://cran.r-project.org/ package $=$ rms. (2018).

20. Chang W., Cheng J., Allaire J., Xie Y. \& McPherson J. shiny: Web Application Framework for R. https://cran.r-project.org/package=shiny. (2018).

21. Kempf, E., Lemoine, N., Tergemina-Clain, G., Turpin, A., Postel-Vinay, S., Lanoy, E. et al. A case-control study brings to light the causes of screen failures in phase 1 cancer clinical trials. PLoS ONE 11, e0154895 (2016).

22. Lui, Y. Y. N. \& Lo, Y. M. D. Circulating DNA in plasma and serum: Biology, preanalytical issues and diagnostic applications. Clin. Chem. Lab. Med. 40, 962-968 (2002).

23. Fleischhacker M. \& Schmidt B. Circulating nucleic acids (CNAs) and cancer-a survey. Biochim. Biophys. Acta. 1775, 181-232 (2007)

24. Devonshire, A. S., Whale, A. S., Gutteridge, A., Jones, G., Cowen, S., Foy, C. A. et al. Towards standardisation of cell-free DNA measurement in plasma: controls for extraction efficiency, fragment size bias and quantification. Anal. Bioanal. Chem. 406, 6499-6512 (2014).

25. Dietz S., Schirmer U., Mercé C., Von Bubnoff N., Dahl E., Meister M., et al. Low input whole-exome sequencing to determine the representation of the tumor exome in circulating DNA of non-small cell lung cancer patients. PLOS ONE 11, e0161012 (2016).

26. Olmos, D., Baird, R. D., Yap, T. A., Massard, C., Pope, L., Sandhu, S. K. et al. Baseline circulating tumor cell counts significantly enhance a prognostic score for patients participating in phase I oncology trials. Clin. Cancer Res. 17, 5188-5196 (2011). 